\title{
Towards Meaning-Oriented Language Modeling
}

\author{
Günther Wirsching \\ Katholische Universität Eichstätt-Ingolstadt \\ Mathematisch-Geographische Fakultät \\ 85072 Eichstätt, Germany \\ Email: guenther.wirsching@ku.de
}

\author{
Robert Lorenz \\ Universität Augsburg \\ Institut für Informatik \\ 86159 Augsburg, Germany \\ Email: robert.lorenz@informatik.uni-augsburg.de
}

\begin{abstract}
Based on a mathematical model for Skinner's functional analysis of Verbal Behavior, we derive formal definitions for the concepts semantic anchor, utterance-meaning pair, and microlocal grammar. We show how these concepts can be used for designing a bidirectional interface connecting automatic speech recognition to meaning oriented language processing. A semiautomatic process for constructing components for such an interface from sparse data, e. g. collected from Wizard of $\mathrm{Oz}$ experiments, is described. Finally, we use our formal approach to investigate some questions concerning the formal complexity of natural language.
\end{abstract}

\section{INTRODUCTION}

For designing human-machine interfaces, it is highly desirable to include the possibility for using spoken language in both directions. To this end, it is necessary that the machine can both: understand human verbal utterances, and formulate agreeable wordings for presenting information, or for asking for specific inputs.

Concerning the first problem, it is also well-known that machines are bad in concept regonition, even if endowed with a speech recognizer with whose word error rate is low. A reason is that in automatic speech recognition, 'speech' and 'language' are often strictly separated. 'Speech analysis' ends with a statistical language model, while 'language analysis' starts with parsing word sequences. Meaning oriented language modeling is a functional approach for creating bidirectional interfaces between speech and meaning.

Basic to our work is a channel model described in the next section, which can be extracted from Skinner's book [1]. The crucial point is Skinner's observation that in stable communication situations, the 'meaning' of a verbal utterance is not part of the utterance itself, but is completely determined by the circumstances of the utterance. For constructing a mathematical model, we employ semantic anchors which provide a connection between Zermelo-Fraenkel set theory with urelements, abbreviated ZFU, and the circumstances of the utterance. This allows us to consider a meaning as a set in ZFU which has some reference to reality in a communicative process. So we arrive at the concept of utterance-meaning pairs, abbreviated UMP, which are the atoms from which a meaning oriented language model is built.

We show that UMP's can be extracted from data collected in appropriate Wizard of $\mathrm{Oz}$ experiments. Based on the UMP concept, we use the notion micro-local grammar for modeling components of a bidirectional interface between speech and language. Moreover, a semi-automatic process for constructing micro-local grammars from sparse data is described.
Finally, our formal approach opens a way for attacking the problem of formal complexity of natural language. We show that it is possible to apply Gödel's theorem and Rice' theorem in our setting, which suggest the conjecture that the UMP's of a natural language are not recursively enumerable.

\section{CHANNEL MODELS}

Mathematical modeling for message transmission starts with Shannon's famous channel model [2], [3]:

$$
\text { Sender } \longrightarrow \text { Channel } \longrightarrow \text { Receiver, }
$$

where a Markov process is employed as mathematical model for transmission. The channel model has proved very useful in modeling transmission of symbol strings, without reference to meaning. Shifting attention to semantics, psychology comes in, as formulation and understanding of messages depends on ontologies and language skills. Similar to Shannon's channel model is Skinner's [1] embedding of verbal behavior into an $A B C$-scheme

Antecedents $\longrightarrow$ Behavior $\longrightarrow$ Consequences,

splitting a communication process into A-, B-, and C-part.

Our first modeling decision here is that we assume that the Behavior is a verbal utterance of bounded length. Moreover, we assume that the verbal utterance is taken from a natural language. It is clear that any fixed natural language only contains a finite number of phonemes, the duration of a phoneme in an utterance is bounded below, and the phrase length is bounded. It follows that, for any fixed natural language, the set of possible utterances is necessarily finite.

As a second modeling decision, we do not consider learning procedures, where iterations are used to change behavior by its consequences. Rather, we concentrate on stable situations, where the behavior has the intention to stimulate or modify consequences. Our aim is to design artificial speech dialogue systems, where the information state of the system should change according to a verbal utterance of an user. Therefore, we take into account only those parts of the C-part which are given by the abilities of the artificial system. We are heading for constructing language models which give a relation between formulations of a human user and updates of the information state of the system.

In laboratory experiments, the ABC-scheme can be used to measure behavior in fixed contexts. In [1], Skinner takes the antecedents and possible consequences as independent variables controlled by the experimentator, and he takes as 
dependent variable the probabilities of different behaviors belonging to fixed $\mathrm{A}$ - and $\mathrm{C}$-parts. Choosing a probability distribution as dependent variable appears quite different from current empirical methods, where it would be more natural to take just the $B$-part as dependent variable, and accept a certain residual variance not explained by the variables under control. For explaining the difference, let us have closer look at Skinner's arguments. Skinner explicitly leaves the question open, whether the B-part is completely determined by the independent variables - in an experimental situation, it is often impossible to control all relevant variables. In fact, A- and Cparts are arranged in such a way that a certain specified number of independent variables are under control of the experimentator. So, the normal situation is that different Behaviors correspond to fixed values of the independent variables. Skinner argues that, in principle, it might be possible that the behavior could be determined if sufficient information on antecendents and (intended) consequences were available-but, as this is not the case in most situations, a probabilistic model appears better suited in a functional-descriptive approach. Provided a sufficiently large number of experiments, such probabilities can be approximated by outcome frequencies. In fact, a probability distribution would be quite a bit more information than mere residual variance.

But where precisely is the 'meaning' of the behavior? Skinner's answer:

Technically, meanings are to be found among the
independent variables in a functional account, rather
than as properties of the dependent variable. When
someone says that he can see the meaning of a
response, he means that he can infer some of the
variables of which the response is usually a function. $[1, \mathrm{p} .14]$

Combining this with the ABC-scheme and Shannon's channel model leads to the following statement: Semantics is a hypothetical intervening variable that links antecedents and behavior on the sender's side, and behavior and consequences an the receiver's side [3].

There is also a system-theoretic approach to semantics, see Bischof [4, Kap. 10], who comes to an essentially equivalent definition of semantics (without reference to Skinner).

\section{MeAning And Language}

\section{A. An Example}

Let us turn to an example. Suppose that we intend to design a human-machine interface based on speech dialogue. For getting best possible performance of the speech recognizer, our aim is to describe the language models configuring the speech recognizer as good as possible. Best performance could be guaranteed if we were able to construct, for each dialogue state separately, a language model containing exactly all possible utterances of users in the given dialogue state, scored by their probabilities of occurence.

For approximating this best possible situation, the ABCscheme (2) is helpful. As described in the previous section, meaning is not part of a verbal behavior, but is to be found in the antecendents $A$ and consequences $C$. So, a meaning is obtained by fixing certain properties of $A$ and $C$-for convenience, we call such a fixed property a (semantic) anchor.

In order to apply this to our speech dialogue example, imagine that somebody, called the commander, gives a flower vase to a servant, and commands the servant in just one utterance $u$ in German language, say, to put the vase at a certain position. According to the preceding paragraph, the meaning of the utterance is to be determined by anchors in the antecedents and consequences. As a minmal set of anchors for the meaning of utterance $u$, consider three anchors $a_{1}, a_{2}, a_{3}$ in $A$, namely the setting giving a situational context $\left(a_{1}\right)$, the assumption that both commander and servant have sufficient knowledge of German language $\left(a_{2}\right)$, that the vase is given to the servant $\left(a_{3}\right)$, and one anchor $c$ in $C$, namely the position where the vase is put to by the servant. The utterance $u$ may contain much more information, e.g., that the servant should carry the vase carefully, or that she or he should avoid some precious carpet when moving.

In this setting, let us consider the set

$$
\mathcal{U}_{1}:=\mathcal{U}\left(\left\{a_{1}, a_{2}, a_{3}, c\right\}\right)
$$

of all utterances whose context fits into an ABC-scheme admitting the given four anchors $a_{1}, a_{2}, a_{3}, c$. If, in addition, we endow each utterance $u \in \mathcal{U}_{1}$ with its relative frequency of occurence $f_{1}(u) \in[0,1]$, the result is a stochastic language model

$$
\mathcal{L}_{1}:=\left(\mathcal{U}_{1}, f_{1}\right)
$$

Each utterance $u \in \mathcal{U}_{1}$ fits, as possible verbal behavior, into an $\mathrm{ABC}$-scheme with anchors $a_{1}, a_{2}, a_{3}, c$, and $f_{1}(u)$ is the conditional probability that, given anchors $a_{1}, a_{2}, a_{3}, c$, utterance $u$ occurs. Of course, this language model would also include utterances containing much more information. In terms of dialogue modeling, $\mathcal{U}_{1}$ would be a language admitting overanswering.

But this situation is not very satisfactory, as $\mathcal{U}_{1}$ does not contain an utterance for commanding the servant carry the vase to another position. For modeling this, split the anchor $c$ into two parts $c_{0}$ and $c_{1}$, where $c_{0}$ means 'put the vase' and $c_{1}$ refers to the position where to put the vase. Now let $c_{2}, c_{3}, \ldots$ refer to other possible positions, and define utterance sets

$$
\mathcal{U}_{i}:=\mathcal{U}\left(\left\{a_{1}, a_{2}, a_{3}, c_{0}, c_{i}\right\}\right)
$$

containing all utterances for commanding the servant to put the vase on position $c_{i}$. The union of the $\mathcal{U}_{i}$ is a language

$$
\mathcal{U}_{0}:=\mathcal{U}\left(\left\{a_{1}, a_{2}, a_{3}, c_{0}\right\}\right):=\bigcup_{i=1,2,3, \ldots} \mathcal{U}_{i}
$$

which contains all take-move-put commands understood by the servant. If we knew relative frequencies $f_{i}(u)$ for utterances $u \in \mathcal{U}_{i}$, and also probabilities $p_{i}$ of occurence of anchor $c_{i}$, we could endow the language $\mathcal{U}_{0}$ with a probability function

$$
f_{0}: \mathcal{U}_{0} \rightarrow[0,1], \quad f_{0}(u):=\sum_{u \in \mathcal{U}_{i}} p_{i} f_{i}(u)
$$

rendering $\mathcal{L}_{0}:=\left(\mathcal{U}_{0}, f_{0}\right)$ a stochastic language model. 


\section{B. Semantic Anchors, Formally Defined}

For working with semantic anchors, it is necessary to define them as mathematical objects—but a semantic anchor must connect natural language to reality. As we argued in section II, any natural language is finite - but it appears misleading to consider reality as finite: for instance, in usual mathematical models, locations and time-points are described by real numbers with arbitrary precision. A third aspect which we incorporate into our formalization is that reality is subjective, i.e., what is considered as real may depend on personal views.

In order to solve these problems, we invoke mathematics as interlingua to bridge the gap between finite natural language and potentially infinite reality. The basis of mathematics is axiomatic set theory. For definiteness, we use Zermelo-Fraenkel's axiomatization including urelements, abbreviated ZFU. In our model, an urelement is an arbitrary reference to a property of reality for at least one of the involved persons. Given the channel model (1), this means that either sender, or receiver, or both, are able to link the urelement to reality. For example, nouns like 'plant' or 'table', verbs like 'take' or 'think', and adjectives like 'nice' or 'terrible' refer to aspects of reality, including inner reality of a person. We take such referencing expressions as urelements - note that, at the moment, we need not assume that the reference is unique or common to different persons.

It is known from mathematics that the language of set theory is very strong. It allows to define any mathematical object, and to express any mathematical assertion. Having added sufficiently many urelements as basic references to reality, it is quite safe to assume that this language is strong enough to express any possible meaning of any verbal behavior.

Let us call an urelement or a set in ZFU a $Z F U$ object. By definition, a semantic anchor is a ZFU object with the additional property that at least one of the involved persons considers it as a description of an aspect or property of reality. We say that a given $\mathrm{ZFU}$ object $e$ is realized in an $A B C$ scheme (2) according to a person $P$, if person $P$ considers $e$ as a description of aspects or properties of antecedents or consequences in the $\mathrm{ABC}$-scheme. We use the formula $R_{P}(e)$ to formally denote the assertion that person $P$ considers $e$ to be realized in the $\mathrm{ABC}$-scheme. Whence assertion $R_{P}(e)$ is true whenever $P$ considers $e$ as a semantic anchor, and $R_{P}(e)$ is false if in $P$ 's view of the world, $e$ is not a semantic anchor.

If a ZFU object contains more than one reference to reality, it is realized in an ABC-scheme iff a person $P$ is able to relate all references to reality simultaneously. That is, combining several references to reality into a single semantic anchor means combining them with logical 'and'. For instance, in the commander-servant context described above, if a ZFU object $s_{1}$ references 'the color of the vase is blue', $s_{2}$ references 'the vase is in the cupboard',

$$
R_{P}\left(\left\{s_{1}, s_{2}\right\}\right)=R_{P}\left(s_{1}\right) \wedge R_{P}\left(s_{2}\right)
$$

formalizes the assertion that 'the blue vase is in the cupboard'. In this case, person $P$ is either the command or the servant. Clearly, effective communication is only possible if the two views coincide, at least concerning the ZFU objects $s_{1}, s_{2}$, and $\left\{s_{1}, s_{2}\right\}$.
The important point of this definition is that ZFU provides a grammar to express any possible semantic anchor, but it is not true that each particular syntactically correct description of a set in ZFU represents a semantic anchor. Examples for ZFU objects which are not semantic anchors are provided by conflicting references to reality. For instance, in the commanderservant context described above, a reference like 'the vase is on the cupboard at time $t_{0}$ ' and a reference like 'the vase is on the table at time $t_{0}$ ' are conflicting. If these two references are encoded into semantic anchors $a_{1}$ and $a_{2}$, respectively, then the ZFU object $\left\{a_{1}, a_{2}\right\}$ is syntactically correct, but not a semantic anchor. Formally, for a given person $P$, two semantic anchors $a_{1}$ and $a_{2}$ are called conflicting w.r.t. $P$, iff the assertion

$$
R_{P}\left(a_{1}\right) \wedge R_{P}\left(a_{2}\right)
$$

is a contradiction.

For applying meaning oriented language modeling to a technical system, we suggest to take the system as 'artificial person', whose world view is given by the data available to the system. There are two techniques especially useful for constructing ZFU objects which can be considered as semantic anchors: the use of feature-values relations, and the use of logical formulae.

1) Feature-Values Relations: According to Definition 1 in [5], a feature-values relation, abbreviated FVR, is a triple

$$
(V, \rightarrow, \ell)
$$

consisting of a finite set $V$ of vertices, a relation $\rightarrow$ on $V$ making $(V, \rightarrow)$ an acyclic directed graph, and a labeling $\ell$ : $V \rightarrow L$, where $L$ is some given set of possible labels. Taking the set of labels from the urelements, it is clear that any FVR can be considered as ZFU object.

In order to get a reference to reality having in mind a speech dialogue system, observe that it is often reasonable to assume that the data available to the system are organized according to an entity-relationship diagram. An algorithm deriving FVR's for referencing sets of entities from the ERdiagram is described in [6].

2) Logical Formulae: Another type of ZFU object which can be part of a semantic anchor are logical formulae. Adopting a calculus for logic, we can consider a logical formula as string of symbols. From this view point, it is possible to take into account arbitrary calculi for logic, including calculi modeling modal or deontic logic.

In order to connect a logical formula to reality, we have to enhance the formula by some construction meaning 'this formula is true in the system's world view', or 'this formula can be inferred from the dialogue up to now', or other formulations in this vein. Such expressions can easily be modeled by urelements.

\section{Utterance-Meaning Pairs}

By definition, an utterance-meaning pair, abbreviated UMP, consists of an utterance, described as finite sequence of words, and a meaning, described as a set of non-conflicting semantic anchors. The notion UMP already has been defined in [7], where only FVR's were admitted as meaning; here we adopt the more general definition using semantic anchors. 
Given a meaning $M$, the set of utterances which can be embedded in an ABC-scheme (2) with meaning $M$ is denoted by $\mathcal{U}(M)$.

Here is a collection of remarks about UMP's:

- The empty set $\emptyset$ is also a meaning, the set $\mathcal{U}(\emptyset)$ contains all possible verbal utterances in any natural language.

- For a fixed natural language, e. g. 'German', we can take the name of the language as urelement. Then the set $\mathcal{U}(\{$ German $\})$ denotes the finite set of all possible German utterances.

- If $M_{1} \subseteq M_{2}$, then $\mathcal{U}\left(M_{1}\right) \supseteq \mathcal{U}\left(M_{2}\right)$.

- A possible meaning $M$ may contain infinitely many semantic anchors, but the set $\mathcal{U}(M)$, being a part of natural language, is necessarily finite.

- Given an utterance $u$, there may be infinitely many possible meanings $M_{i}$ with $u \in \mathcal{U}\left(M_{i}\right)$. An example is the utterance 'ok', whose meaning depends on the context, and belongs to infinitely many different meanings.

\section{A Hierarchy Of Grammars}

According to our modeling assumptions given in II, we know that any natural language is finite. Hence, for any given meaning $M$ containing a reference to a fixed natural language, the set of utterances $\mathcal{U}(M)$ is also finite, which implies that each $\mathcal{U}(M)$ can be described by a regular grammar.

For designing speech dialogue systems, it appears natural to consider two-level semantics as described in [3], where the first level has been called scheme, and the second level has been called specification. This structure can be incorporated into our approach based on semantic anchors as follows. We reserve a sequence $x_{1}, x_{2}, \ldots$ of urelements with no reference to reality as placeholders. A set of ZFU objects containing some placeholders, $x_{1}, \ldots, x_{n}$, say, is called a semantic scheme; let $S:=S\left(x_{1}, \ldots, x_{n}\right)$ be such a scheme. If we replace each placeholder $x_{i}$ by a semantic anchor $a_{i}$, we obtain a ZFU object $S\left(a_{1}, \ldots, a_{n}\right)$, called a specification of the scheme $S$. Note that, at the moment, we do not postulate that the specification is a meaning-it may happen that there is no person who connects the specification to reality, or, in other words, there may be no person $P$ such that $R_{P}\left(S\left(a_{1}, \ldots, a_{n}\right)\right)$ is true.

For example, consider a semantic anchor given by an FVR $(V, \rightarrow, \ell)$. If we replace some of the labels (which are urelements) by placeholders, we get semantic scheme.

The next step is to define the set of utterances belonging to a given semantic scheme $S=S\left(x_{1}, \ldots, x_{n}\right)$. To this end, denote by $A(S)$ the set of all $n$-tuples $\left(a_{1}, \ldots, a_{n}\right)$ of semantic anchors rendering $S\left(a_{1}, \ldots, a_{n}\right)$ a meaning. Then the set of utterances belonging to the semantic scheme $S$ is defined as the union

$$
\mathcal{U}(S):=\bigcup_{\left(a_{1}, \ldots, a_{n}\right) \in A(S)} \mathcal{U}\left(S\left(a_{1}, \ldots, a_{n}\right)\right) .
$$

In computational linguistics, a grammar describing phrases belonging to meanings from some specific domain is often called a local grammar. This leads to a chain of concepts

$$
\text { domain - semantic scheme - meaning. }
$$

The UMP concept allows us to convert this chain into a hierarchy of grammars. Explicitly, fix a natural language by a semantic anchor $L$, and assume that a domain in language $L$ is given by a set $D$ of semantic anchors, with $L \in D$. Then $\mathcal{U}(D)$ is the set of utterances belonging to domain $D$. It is a finite set, whence it can be described by a grammar $\Gamma(D)$, the local grammar of language $L$ pertaining to domain $D$. Now fix a meaning $M$ inside the domain $D$, which means $M \supseteq D$. We call a grammar $\Gamma(M)$ describing the utterance set $\mathcal{U}(M) \subseteq \mathcal{U}(D)$ an elementary grammar, or single-meaning grammar. Replacing some of the semantic anchors in $M \backslash D$ by placeholders $x_{1}, \ldots, x_{n}$, we arrive at a semantic scheme $S=S\left(x_{1}, \ldots, x_{n}\right) \supseteq D$ with the property that $M$ is a specification of $S$. In view of (3), this gives a chain of sets in ZFU

$$
\mathcal{U}(D) \supseteq \mathcal{U}(S) \supseteq \mathcal{U}(M) .
$$

In this situation, we call a grammar describing the utterance set $\mathcal{U}(S)$ defined in (3) a micro-local grammar. Then the result is a hierarchy of grammar adjectives reflecting the chain (4):

$$
\text { local-micro-local-elementary (grammar). }
$$

Formally, as we specified a domain by a set of semantic anchors, a local grammar is a special case of an elementary grammar. In the next subsection we will see that, from a practical point of view, it is an advantage to have the intermediate concept micro-local grammar based on placeholders for semantic anchors.

\section{E. Skinner's ABC-Scheme Revisited}

Now let us return to Skinner's ABC-scheme (2). In section II we argued that Skinner's dependent variable is a probability distribution on the set of possible utterances fitting into $\mathrm{ABC}$-schemes where certain variables in the $\mathrm{A}$ - and $\mathrm{C}$ parts are fixed. Using our UMP concept, we can give more precision to this statement. If $M$ is a meaning, i. e., a set of semantic anchors, the utterances set $\mathcal{U}(M)$ describes the possible B-parts of ABC-scheme realizing $M$. In this setting, Skinner's dependent variable is a probability function

$$
f: \mathcal{U}(M) \rightarrow[0,1]
$$

In other words, given a meaning $M$, Skinner's dependent variable is a stochastic language model for the possible utterances belonging to meaning $M$,

$$
\mathcal{L}(M):=(\mathcal{U}(M), f) .
$$

As mentioned in section II, with sufficient experimental data fitting into $\mathrm{ABC}$-schemes realizing $M$, we could derive an approximation to $\mathcal{L}(M)$.

In principle, Wizard of $\mathrm{Oz}$ experiments can be designed in such a way that certain sets of semantic anchors pertaining to certain specified semantic schemes can be realized. See [8] for a Wizard of $\mathrm{Oz}$ experiment imitating a home entertainment system, which is designed using the UMP concept. A problem is the small amount of data collected by such a Wizard of $\mathrm{Oz}$ 
experiment. Given a meaning $M$, we get only view utterances belonging to $M$, if any-indeed, most meanings which are possible by the system are not represented in an utterance. Technically, this sparse data problem means that in most cases there not sufficient data for a direct construction of a language model $\mathcal{L}(M)$ as defined in (7).

In view of the sparse data problem, our first decision is to take the probability function as two-valued. Consequently, $f$ is a scalar multiple af a characteristic function. In this model, there is subset $H \subseteq \mathcal{U}(M)$ such that the utterance in $\mathcal{U} \backslash H$ are neglected, and all utterances in $H$ get the same probability. Our second decision is motivated by the fact that many possible meanings may never occur in our experiments. Therefore, we concentrate on semantic schemes with placeholders rather than specific meanings.

\section{F. Construction Of Meaning Oriented Language Models}

Our aim is to construct, given a semantic scheme $S$, an approximation to the utterance set $\mathcal{U}(S)$ defined in (3) from sparse data, which is as good as possible from the data.

Given an UMP $(u, M)$, and a semantic scheme $S$ with placeholders $x_{1}, \ldots, x_{n}$ such that $M$ is a specification of $S$. Then there are semantic anchors $a_{1}, \ldots, a_{n}$ such that $M=$ $S\left(a_{1}, \ldots, a_{n}\right)$. The idea is to employ linguistic knowledge for locating words corresponding to the semantic anchors in the utterance $u$. The next step is to replace these words by others referring to other semantic anchors, expanding $u$ to a set $B(u, S)$ of utterances, where the letter $B$ refers to the B-part of an ABC-scheme. We infer from (3) that $B(u, S) \subseteq \mathcal{U}(S)$.

For illustrating this procedure let us modify and extend our example started in subsection III-A. It is clear that the semantic anchors $a_{1}, a_{2}, a_{3}, c$ described there can, in principle, be formalized as ZFU objects. The modification is that the servant is not given the object, but has to take the object from where it is, and our plan is to construct a language model which enables the servant to conclude from the utterance which object he should take for moving. So we modify semantic anchor $a_{3}$ to mean 'take the flower vase', and we add a further sematic anchor $a_{4}$ meaning 'take the ashtray'.

In this example, given the semantic scheme

$$
S=\left\{a_{1}, a_{2}, x, c\right\}
$$

and the utterance

$$
u=\text { "Stell' die Blumenvase auf die Kommode!" }
$$

the following process will lead to a set $B(u, S)$ :

1) Identify in $u$ the parts relating $u$ to semantic anchors which replace placeholders in $S$ : here $x$ corresponds to "die Blumenvase".

2) Use the database to identify all tuples which lead to specification of $S$ which are meanings: here $x$ can be specified to "Blumenvase" or "Aschenbecher".

3) Create utterances like $u$ which belong to the meanings identified in the second step: in this example, we get

$$
B(u, S)=\left\{\left(u,\left\{a_{1}, a_{2}, a_{3}, c\right\}\right),\left(\tilde{u},\left\{a_{1}, a_{2}, a_{4}, c\right\}\right)\right\}
$$

with the utterance

$$
\tilde{u}=\text { "Stell' den Aschenbecher auf die Kommode!" }
$$

Note that for performing the first and the third step of this procedure, linguistic knowledge is essential: In order to come to appropriate wordings, it is necessary to insert the German articles in their correct genders and cases.

Let $u_{1}, \ldots, u_{n}$ be the utterances collected in a Wizard of $\mathrm{Oz}$ experiment, and let $S_{1}, \ldots, S_{m}$ denote the different semantic schemes available in the world view of the system. Then there is a many-to-many relation

$$
R_{B} \subseteq\left\{u_{1}, \ldots, u_{n}\right\} \times\left\{S_{1}, \ldots, S_{m}\right\}
$$

defined by

$$
\left(u_{i}, S_{k}\right) \in R_{B} \quad: \Leftrightarrow \quad u_{i} \text { belongs to } S_{k} .
$$

Using the $u_{i}$ as described above, we are now able to construct sets

$$
\mathcal{B}\left(S_{k}\right):=\bigcup_{\left(u_{i}, S_{k}\right) \in R_{B}} B\left(u_{i}, S_{k}\right) .
$$

Note that, in order to construct these sets, we need at least one utterance for each semantic scheme $S_{k}$.

\section{G. Using Meaning Oriented Language Models}

In a speech dialogue system, meaning oriented language modeling can be used for configuring the speech recognizer at each dialogue state separately. Such a system would contain a number of semantic schemes $S_{1}, \ldots, S_{m}$ modeling the various contexts which might occur during a human-machine dialogue. In addition, for each $S_{k}$, the system would contained a precompiled micro-local grammar describing $\mathcal{B}\left(S_{k}\right)$. At each dialogue state, the system would choose all semantic schemes which fit into the given context. Then the chosen semantic schemes are activated by loading their corresponding precompiled microlocal grammars into the speech recognizer.

Note that we do not assume that the sets $\mathcal{B}\left(S_{k}\right)$ are pairwise disjoint. If an utterance happens to belong to two different activated semantic schemes, in many cases the ambiguity will be either not so important or solved when the dialogue procedes.

\section{H. A Remark About Bidirectionality}

For application in human-machine communication, it is desirable to have a speech-meaning interface which is bidirectional. The machine should be able to provide data, or to pose a question, using agreeable verbal utterances.

Given a semantic scheme $S$, the construction described in subsection III-F produces a map defined on $\mathcal{U}(S)$, which maps each utterance meaning pair $(u, M)$ belonging to $S$ to a subset $B(u, S) \subseteq \mathcal{U}(S)$. Moreover, the map

$$
(u, M) \quad \longmapsto \quad(B(u, S), M)
$$

is one-to-one. This enables the machine to select an appropriate utterance $u^{\prime}$ as follows. Given a set $B(u, S)$ and a meaning $M^{\prime}$ pertaining to $S$, there is a uniquely determined utterance $u^{\prime}$ such that $\left(u^{\prime}, M^{\prime}\right) \in B(u, S)$. 


\section{ON The Formal Complexity Of NATURAL LANGUAGE}

The formal construction of semantics given in this article is a starting point for attacking the problem of formal complexity of natural language. In section II we argued that, if a natural language is considered as set of verbal utterances, it is necessarily finite. Essentially, this is due to the boundedness of phrase length.

But if we consider a natural language as consisting of utterance-meaning pairs, the situation changes dramatically. Contrary to phrase length, there is no obvious bound for the number of semantic anchors. Indeed, it is conceivable that an utterance belongs to a meaning containing infinitely many semantic anchors. Hence, in general, the boundedness argument is not applicable. In this context, the problem of formal complexity arises.

According to our definition given in subsection III-B, a semantic anchor is a ZFU object which some person considers as description of an aspect or property of reality. Given the language and rules of set theory, and provided the urelements are enumerable, it is clear that the class of ZFU objects is recursively enumerable. But not all ZFU objects are semantic anchors. Our conjecture is the following.

CONJECTURE. Given a person $P$ who considers the arithmetic of natural numbers as an aspect of reality, and who satisfies certain mild consisteny requirements, then the class of semantic anchors of this person is not recursively enumerable.

Unfortunately, we do not have a formal proof of this conjecture. But we have some results in this direction.

\section{A. Application of Gödel's Theorem}

Assume that a person $P$ admits an urelement

$$
T=\text { "mathematically true" }
$$

such that an arithmetic assertion $a$ is true, if and only if $P$ considers $(T, a)$ as semantic anchor. In this situation, Gödel's famous Incompleteness Theorem implies that the subset of semantic anchors consisting of ZFU objects of the type $(T, a)$, where $a$ is a valid arithmetic assertion, is not recursively enumerable.

Let us additionally assume that $P$ accepts the urelement $T$ only as part of a semantic anchor if appears in the form $(T, a)$ where $a$ is arithmetic assertion. Then we conclude that the subclass of semantic anchors of $P$ which contains the urelement $T$ is not recursively enumerable. This does not necessarily imply that the class of all semantic anchors for $P$ is not recursively enumerable.

\section{B. Application of Rice' Theorem}

Recall from subsection III-C that a meaning is a set of semantic anchors which is itself a semantic anchor. We now assume that a person $P$ admits a countable set of basic semantic anchors $B=\left\{b_{1}, b_{2}, b_{3}, \ldots\right\}$ containing at least one conflicting pair $\left(b_{i}, b_{k}\right)$, with $i<k$, say. Denote by $\mathcal{M}$ the set of possible meanings based on $B$, i. e., the set of subsets $M \subseteq B$ such that $\mathcal{U}(M) \neq \emptyset$. As semantic anchors without utterance could be omitted, it is safe to assume that all singletons $\left\{b_{i}\right\}$ are possible meanings. Identifying $B$ with the set $\mathcal{N}$ of natural numbers, we infer that $\mathcal{M}$ can be identified with a class of subsets of $\mathcal{N}$.

In [9], Rice considers arbitrary classes $A$ of subsets of $\mathcal{N}$. For such classes he introduces the notion completely recursively enumerable, abbreviated c.r.e. Here we dispense with the comparatively complicate formal definition of c.r.e. classes. Intuitively, a class $A$ is c.r.e., iff the set of all partially recursive functions which enumerate a set in $A$ is recursively eumerable. The crux is that, in general, there are infinitely many different functions enumerating recursively a given set. Rice proves that only classes of special type are c.r.e., and 'most' classes are not. He uses these results to infer computability results of certain subsets of $\mathcal{N}$.

We are able to apply Rice' main theorem for proving that the class $\mathcal{M}$ is not c.r.e. There is no direct use of this result, but, as indicated above, there is a subtle connection between c.r.e. classes and recursively enumerable subsets of $\mathcal{N}$. Therefore, the result might be a starting point for further inverstigations. Rice stated his main theorem as follows [9, p. 363]:

\section{THEOREM 6. Let A be a class which contains a finite set $\left\{a_{0}, \ldots, a_{k}\right\}$, but omits a set a which includes $\left\{a_{0}, \ldots, a_{k}\right\}$. Then $A$ is not c.r.e..}

Indeed, it follows from the assumption that all singletons are possible meanings that $\mathcal{M}$ contains the set $\left\{b_{i}\right\}$. As the semantic anchors $b_{i}$ and $b_{k}$ are conflicting, it follows that $\mathcal{M}$ omits the set $\left\{b_{i}, b_{k}\right\}$. Consequently, the class $\mathcal{M}$ of possible meanings is not c.r.e.

\section{REFERENCES}

[1] B. F. Skinner, Verbal Behavior. Prentice-Hall, Englewood Cliffs, New Jersey, 1957

[2] C. Shannon, "A mathematical theory of communication," The Bell System Technical Journal, vol. 27, pp. 379-423 and 623-656, 1948.

[3] G. Wirsching, "Calculating semantic uncertainty," in 2012 IEEE 3rd International Conference on Cognitive InfoCommunication, Košice, Slovakia, December 2-5, 2012, 2012, pp. 71-76.

[4] N. Bischof, Struktur und Bedeutung: eine Einführung in die Systemtheorie für Psychologen, 2nd ed. Bern: Huber, 1998.

[5] G. Wirsching, M. Huber, C. Kölbl, R. Lorenz, and R. Römer, "Semantic dialogue modeling," in Behavioral Cognitive Systems, ser. LNCS, A. Esposito, A. M. Esposito, A. Vinciarelli, R. Hoffmann, and V. C. Müller, Eds., vol. 7403, 2012, pp. 104-113.

[6] M. Huber, C. Kölbl, R. Lorenz, R. Römer, and G. Wirsching, "Semantische Dialogmodellierung mit gewichteten Merkmal-Werte-Relationen," in Elektronische Sprachsignalverarbeitung 2009. Tagungsband der 20. Konferenz. Dresden, 21. bis 23. September 2009, ser. Studientexte zur Sprachkommunikation, R. Hoffmann, Ed., vol. 53. TUDpress, Sep. 2009, pp. 25-32.

[7] G. Wirsching and C. Kölbl, "Language modeling with utterancemeaning pairs," Angewandte Informatik, Universität Augsburg, Tech. Rep. 2011-12, 2011. [Online]. Available: http://www.informatik.uniaugsburg.de/lehrstuehle/inf/ publikationen/reports/TR2011-12/

[8] K. Karnagel, R. Römer, S. Rogge, and J. Lindemann, "Semantische Modellierung von System-Benutzer-Interaktionen am Beispiel einer Home-Enterteinment Steuerung," in ESSV2012, ser. Studientexte zur Sprachkommunikation, M. Wolff, Ed., vol. 64. Dresden: TUDPress, August 2012, pp. 306-313.

[9] H. G. Rice, "Classes of recursively enumerable sets and their decision problems," Transactions of the American Mathematical Society, vol. 74, pp. 353-366, 1953. 